\title{
Galaxy Clustering and Baryon Acoustic Oscillations
}

\author{
B. Hoeneisen* ${ }^{\dagger}$ \\ Universidad San Francisco de Quito, Quito, Ecuador \\ E-mail: bhoeneiseneusfq.edu.ed
}

\begin{abstract}
We present measurements of Baryon Acoustic Oscillation (BAO) distances used as an uncalibrated standard ruler that determine $\Omega_{\mathrm{de}}(a), \Omega_{k}, \Omega_{m}$, and $d_{\mathrm{BAO}} \equiv r_{*} H_{0} / c$; and BAO distances used as a calibrated standard ruler $r_{*}$ that constrains a combination of $\sum m_{v}, h$, and $\Omega_{b} h^{2}$. The cosmological parameters obtained in this analysis are compared with the Review of Particle Physics, PDG 2018.
\end{abstract}

2nd World Summit: Exploring the Dark Side of the Universe 25-29 June, 2018

University of Antilles, Pointe-Ãă-Pitre, Guadeloupe, France

\footnotetext{
* Speaker.

${ }^{\dagger}$ I thank the organizers of the Guadeloupe Conference for the kind invitation.
} 


\section{Introduction}

At the Guadeloupe "2nd World Summit on: Exploring the Dark Side of the Universe" 2018 were presented several experiments that measure combinations of correlated cosmological parameters. The problem is how to extract the cosmological parameters from these measurements (that show mild tensions). The precise measurements of fluctuations of the Cosmic Microwave Background $(\mathrm{CMB})$ temperature across almost the entire sky by the Planck experiment allows independent and precise determinations of the base set of six cosmological parameters [1] under these assumptions: (i) space is flat, i.e. curvature $\Omega_{k}=0$, (ii) the dark energy density is constant, i.e. $\Omega_{\mathrm{de}}(a)=\Omega_{\Lambda}$ is independent of the expansion parameter $a$, and (iii) neutrino masses are negligible. At the Guadeloupe meeting I presented measurements of Baryon Acoustic Oscillation (BAO) distances used as an uncalibrated standard ruler that address (i) and (ii), and as a calibrated standard ruler that constrains a combination of $\sum m_{v}, h$, and $\Omega_{b} h^{2}$. (We use the standard notation of the Review of Particle Physics, PDG 2018 [纤). $\sum m_{v}$ is the sum of masses of three active neutrino eigenstates assumed to be nearly degenerate, i.e. $\sum m_{v} \approx 3 m_{v}$. In the following, I will comment on these measurements, and on some minor tensions discussed at the Guadeloupe meeting. Neutrino masses measured with the Sachs-Wolfe effect, $\sigma_{8}$, and the galaxy power spectrum $P_{\text {gal }}(k)$ are discussed in a separate contribution to this conference. For details I refer the reader to the Guadeloupe talks [3], and to the publications [4], [5], [6], and references therein.

\section{BAO as an uncalibrated standard ruler}

From the acoustic sound horizon angle $\theta_{\mathrm{MC}}$ accurately measured by the Planck experiment, and 18 galaxy BAO distance measurements with Sloan Digital Sky Survey SDSS DR13 galaxies in the red shift range 0.1 to 0.7 , we obtain $\Omega_{k}, \Omega_{\mathrm{de}}(a)+2.2 \Omega_{k}$, and the adimensional standard ruler length $d_{\mathrm{BAO}} \equiv r_{s}^{\prime} H_{0} / c$, where $r_{s}^{\prime} \equiv r_{*}$ is the comoving size of the sound horizon [ $₫$. The results are presented in Table 2.1 for several scenarios. Note that $\Omega_{k}$ is consistent with zero, and $\Omega_{\mathrm{de}}(a)$ is consistent with being independent of the expansion parameter $a$. Note also that the constraint on $\Omega_{k}$ becomes tighter if $\Omega_{\mathrm{de}}(a)$ is assumed constant, and that the constraint on $\Omega_{\mathrm{de}}(a)$ becomes tighter if $\Omega_{k}$ is assumed zero. From now on, we assume that $\Omega_{\mathrm{de}}(a) \equiv \Omega_{\Lambda}$ is constant, and $\Omega_{k}=0$. With these assumptions we obtain from Table 1

$$
\Omega_{\Lambda}=0.719 \pm 0.003, \quad d_{\mathrm{BAO}}=0.0340 \pm 0.0002, \quad \Omega_{m}=0.281 \pm 0.003 .
$$

All uncertainties are at $68 \%$ confidence with the listed assumptions. These results are robust because they are independent of any other cosmological parameter (in particular are independent of $h$, and are independent of $\sum m_{v}$ at the present level of accuracy), and depend on theory only through the Friedmann equation (which defines $\Omega_{k}, \Omega_{\Lambda}$, and $\Omega_{m}$ ). The main difficulty with these $\mathrm{BAO}$ measurements is the low significance of the BAO signals due to cosmological fluctuations, so that many redundant measurements were made as emphasized in the Guadeloupe talk. In particular, we measure separately $\hat{d}_{z}$ for galaxy pairs approximately along the line of sight, $\hat{d}_{\alpha}$ for

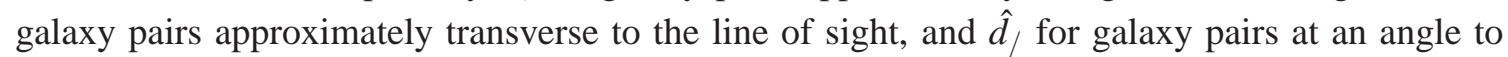
the line of sight 㺻. Measuring the independent BAO lengths $\hat{d}_{z}, \hat{d}_{\alpha}$, and $\hat{d}_{/}$, allows a consistency check $Q=\hat{d}_{/} /\left(\hat{d}_{\alpha}^{0.57} \hat{d}_{z}^{0.43}\right)=1$, and also provides a direct measurement of the uncertainties. See 


\begin{tabular}{c|cccc}
\hline & Scenario 1 & Scenario 1 & Scenario 4 & Scenario 4 \\
\hline$\Omega_{k}$ & 0 fixed & $0.002 \pm 0.007$ & 0 fixed & $-0.015 \pm 0.030$ \\
$\Omega_{\mathrm{de}}+2.2 \Omega_{k}$ & $0.719 \pm 0.003$ & $0.718 \pm 0.004$ & $0.718 \pm 0.004$ & $0.717 \pm 0.004$ \\
$w_{1}$ & n.a. & n.a. & $0.06 \pm 0.15$ & $0.37 \pm 0.61$ \\
$100 d_{\mathrm{BAO}}$ & $3.40 \pm 0.02$ & $3.39 \pm 0.02$ & $3.39 \pm 0.03$ & $3.37 \pm 0.05$ \\
$\chi^{2} /$ d.f. & $11.2 / 17$ & $11.2 / 16$ & $11.1 / 16$ & $10.8 / 15$ \\
\hline
\end{tabular}

Table 1: Cosmological parameters obtained from 18 galaxy BAO measurements with SDSS DR13 galaxies plus $\theta_{\mathrm{MC}}=0.010410 \pm 0.000005$ from the Planck experiment in several scenarios 㺻. Corrections for peculiar motions have been applied. Scenario 1 has $\Omega_{\mathrm{de}}(a)$ constant. Scenario 4 has $\Omega_{\mathrm{de}}(a)=$ $\Omega_{\mathrm{de}}\left[1+w_{1}(1-a)\right]$.

Figure 1. Simulations show [7] that an initial point-like peak in the density, results in concentric shells of overdensity of radius $\approx 148 \mathrm{Mpc}$ and $\approx 18 \mathrm{Mpc}$. The observed BAO signal extends from $\approx(148-11) \mathrm{MpcH}_{0} / c \approx 0.0314$ to $\approx(148+11) \mathrm{MpcH}_{0} / c \approx 0.0365$ as shown in Figure 1 . The only (relatively small) correction applied is for peculiar motions.
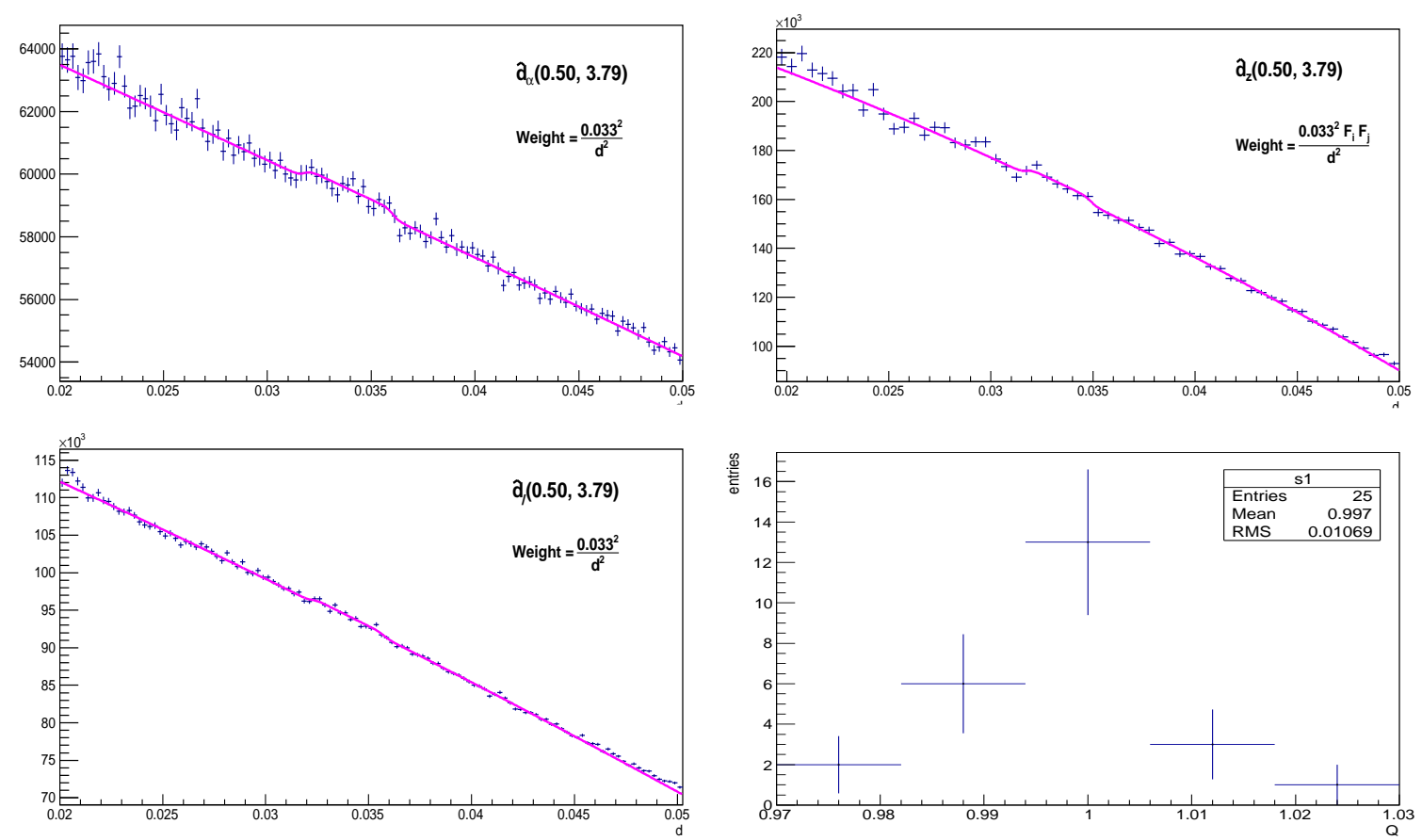

Figure 1: Fits to histograms of galaxy-galaxy distances $d$, in units of $c / H_{0}$, that obtain the BAO distances $\hat{d}_{\alpha}, \hat{d}_{z}$, and $\hat{d}_{/}$, and distribution of the consistency parameter $Q=\hat{d}_{/} /\left(\hat{d}_{\alpha}^{0.56} \hat{d}_{z}^{0.44}\right)=1$.

\section{A comment on $d_{\text {drag }}$ and $d_{*}$}

From the 18 galaxy BAO distance measurements alone, i.e. without the sound horizon angle $\theta_{\mathrm{MC}}$, we obtain the length of the standard ruler in units of $c / H_{0}: d_{\mathrm{drag}} \equiv r_{\mathrm{drag}} H_{0} / c=0.0339 \pm$ 0.0002 , and $\Omega_{m}=0.284 \pm 0.014$, see Table 4 of [ [ $]$ ]. Adding 2 Lyman-alpha measurements obtains $d_{\text {drag }}=0.0340 \pm 0.0002$ and $\Omega_{m}=0.278 \pm 0.011$. The comoving size of the sound horizon is 


\begin{tabular}{l|l|l}
\hline & This analysis & PDG 2018 \\
\hline$\Omega_{k}$ & $0.002 \pm 0.007$ & $-0.005_{-0.009}^{+0.008}$ \\
\hline$\Omega_{\Lambda}$ & $0.719 \pm 0.003$ & $0.692 \pm 0.012$ \\
$\Omega_{m}$ & $0.281 \pm 0.003$ & $0.308 \pm 0.012$ \\
$d_{\mathrm{BAO}}$ & $0.0340 \pm 0.0002$ & \\
\hline$h+0.0255 \cdot \sum m_{v} / \mathrm{eV}$ & $0.6970 \pm 0.0054$ & \\
$h$ & $0.691 \pm 0.012$ & $0.678 \pm 0.009$ \\
\hline
\end{tabular}

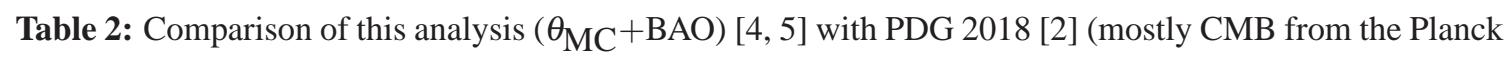
Collaboration (2015)+BAO). $\Omega_{\mathrm{de}}(a)=\Omega_{\Lambda}$ is assumed constant. Curvature $\Omega_{k}$ is assumed zero, except in first row. The first 4 rows of this analysis are from uncalibrated BAO (including $\theta_{\mathrm{MC}}$ ), while the last two are from calibrated BAO with $z_{*}=1089.9 \pm 0.4$.

$r_{*}$. We obtain $d_{*} \equiv r_{*} H_{0} / c \equiv \theta_{\mathrm{MC}} \chi\left(\Omega_{m}, z_{*}\right)=(0.03402 \pm 0.00002) \cdot\left(0.28 / \Omega_{m}\right)^{0.4}$ with $\theta_{\mathrm{MC}}=$ $0.010410 \pm 0.000005$ measured by the Planck experiment. $\chi\left(\Omega_{m}, z_{*}\right) c / H_{0}$ is the angular diameter distance at decoupling. For $\Omega_{m}=0.281 \pm 0.003$ we obtain $r_{*} H_{0} / c=0.03397 \pm 0.00016$. We conclude that the measured $r_{\mathrm{drag}}$ and $r_{*}$ are equal within the quoted uncertainties. For this reason we have set $r_{\mathrm{drag}}=r_{*}$ and $d_{\mathrm{drag}}=d_{*} \equiv d_{\mathrm{BAO}}$. Any uncertainty due to $r_{\mathrm{drag}} \neq r_{*}$ is not included in (2.1).

\section{BAO as a calibrated standard ruler}

The calibrated standard ruler length $r_{*}$ is obtained by integrating the photon-electron-baryon plasma sound speed from primordial time until decoupling at $z_{*}=1089.9 \pm 0.4$ [2]. From $d_{\mathrm{BAO}} \equiv$ $r_{*} H_{0} / c=0.0340 \pm 0.0002$ we obtain a constraint on a combination of $\sum m_{v}, h$ and $\Omega_{b} h^{2}$. Reference [5] takes $z_{\text {drag }}=z_{*}=1089.9 \pm 0.4$ and obtains

$$
\sum m_{v}=0.711-0.335 \cdot \delta h+0.050 \cdot \delta b \pm 0.063 \mathrm{eV}
$$

and $\Omega_{m}=0.282 \pm 0.003$, where $\delta h \equiv(h-0.678) / 0.009$, and $\delta b \equiv\left(\Omega_{b} h^{2}-0.02226\right) / 0.00023$. Alternatively, from $r_{*} H_{0} / c \equiv \theta_{\mathrm{MC}} \chi\left(\Omega_{m}, z_{*}\right)$ we obtain

$$
\Omega_{m}^{0.32}\left(h+0.0255 \sum m_{v} / \mathrm{eV}\right) \cdot\left(\Omega_{b} h^{2}\right)^{-0.19}=0.9551 \pm 0.0006 .
$$

From $\Omega_{m}=0.281 \pm 0.003$ and $\Omega_{b} h^{2}=0.0225 \pm 0.0008$ at $68 \%$ confidence from Big Bang Nuleosynthesis [2], and allowing $0.06<\sum m_{v}<0.68 \mathrm{eV}$ [纤], we obtain from (4.1] or (4.2):

$$
h+0.0255 \cdot \sum m_{v} / \mathrm{eV}=0.6970 \pm 0.0054, \quad \text { and } \quad h=0.691 \pm 0.012 .
$$

\section{Conclusions}

The cosmological parameters obtained in this analysis are compared with [2] in Table 2. Constraints on neutrino masses are presented in the companion talk and article in these proceedings [3]. 


\section{References}

[1] Planck Collaboration: N. Aghanim et al. , arXiv:1807.06209 (2018)

[2] M. Tanabashi et al. (Particle Data Group), The Review of Particle Physics (2018), Phys. Rev. D 98, 030001 (2018)

[3] Guadeloupe 2018 meeting talks: https://indico.cern.ch/event/645015/timetable/\#20180625

[4] B. Hoeneisen, (2017) Study of Baryon Acoustic Oscillations with SDSS DR13 Data and Measurements of $\Omega_{k}$ and $\Omega_{\mathrm{DE}}(a)$. International Journal of Astronomy and Astrophysics, 7, 11-27. https://doi.org/10.4236/ijaa.2017.71002

[5] B. Hoeneisen, (2018) Constraints on Neutrino Masses from Baryon Acoustic Oscillation Measurements. International Journal of Astronomy and Astrophysics, 8, 1-5. https://doi.org/10.4236/ijaa.2018.81001

[6] B. Hoeneisen, (2018) Study of galaxy distributions with SDSS DR14 data and measurement of neutrino masses, arXiv:1806.01227, to be published in International Journal of Astronomy and Astrophysics

[7] D. J. Eisenstein, H.-J. Seo, and M. White, ApJ, 664: 660-674 (2007). 